\title{
Suppressive role of $\mathrm{miR}-502-5 \mathrm{p}$ in breast cancer via downregulation of TRAF2
}

\author{
LI-LI SUN ${ }^{1}$, JIAN WANG ${ }^{1}$, ZHI-JUAN ZHAO ${ }^{1}$, NING LIU $^{2}$, AI-LIAN WANG ${ }^{1}$, HUA-YAN REN ${ }^{1}$, \\ FAN YANG $^{1}$, KE-XIN DIAO $^{1}$, WEI-NENG FU ${ }^{3}$, EN-HUA WANG ${ }^{1}$ and XIAO-YI MI ${ }^{1}$ \\ ${ }^{1}$ Department of Pathology, First Affiliated Hospital and College of Basic Medical Science, China Medical University; \\ ${ }^{2}$ Department of Pancreatic Surgery, First Affiliated Hospital, China Medical University; \\ ${ }^{3}$ Department of Medical Genetics, China Medical University, Shenyang, Liaoning 110001, P.R. China
}

Received December 27, 2013; Accepted February 7, 2014

DOI: $10.3892 /$ or.2014.3105

\begin{abstract}
TRAF2 promotes cancer cell survival, proliferation and metastasis through the NF- $\mathrm{NB}$ pathway by directly interacting with various TNF recepors. However, the molecular mechanism of TRAF2 dysregulation in breast cancer remains to be elucidated. In the present study, miR-502-5p was predicted as a potential regulator of TRAF2. miR-502-5p was significantly downregulated in breast cancer tissues when compared to the level in paired normal breast tissues. The breast cancer cell lines including MCF-7 and MDA-MB-231 expressed a lower level of miR-502-5p when compared to the level in the non-malignant breast epithelial cell line MCF-10A. In vitro, miR-502-5p enhanced early apoptosis and inhibited proliferation of breast cancer cells. Luciferase reporter assay results showed that miR-502-5p could bind to the 3'-untranslated region of the TRAF2 gene, thus, exerting an inhibitory effect on TRAF2. Furthermore, silencing of TRAF2 exhibited effects similar to those of exogenous miR-502-5p, while overexpression of TRAF2 partially abrogated miR-502-5p-mediated suppression in breast cancer cells. In conclusion, miR-502-5p may act as a tumor-suppressor gene by targeting oncogenic TRAF2 in breast cancer and, therefore, may be a potential diagnostic and anticancer therapeutic marker for breast cancer.
\end{abstract}

\section{Introduction}

The tumor necrosis factor receptor-associated factor (TRAF) family, including seven protein members (TRAF1-7), has emerged as the major signal transducers for the TNF receptor and interleukin-1 receptor/Toll-like receptor (IL-1R/TLR)

Correspondence to: Professor Xiao-Yi Mi, Department of Pathology, First Affiliated Hospital and College of Basic Medical Science, China Medical University, 92 Beier Road, Heping, Shenyang, Liaoning 110001, P.R. China

E-mail: mixiaoyi2005@163.com

Key words: apoptosis, breast cancer, TRAF2, miR-502-5p, proliferation superfamilies. TRAFs collectively play a pivotal role in diverse biological processes, including immunity, inflammation and apoptosis $(1,2)$. TRAF2 is unique as an adaptor protein among the TRAF family members. Numerous studies have shown that TRAF2 is a critical mediator of NF- $\kappa \mathrm{B}(3-8)$, JNK and p38 pathway activation $(6,9,10)$, and overexpression of the native TRAF2 gene can activate JNK, p38 and NF- $\kappa$ B in the absence of extracellular stimuli $(7,9,11,12)$.

microRNAs (miRNAs) are a class of small non-coding RNAs, 18-25 nucleotides in length, which play important roles in post-transcriptional gene expression. By binding to complementary sequences predominantly found in the 3'-UTR of target mRNAs, miRNAs block the translation or decrease the stability of mRNAs (13-15). More than $60 \%$ of human proteincoding genes are under selective pressure to maintain pairing to miRNAs, suggesting that most mammalian mRNAs are conserved targets of miRNAs (16). miRNAs are involved in a variety of biological processes including tumor cell proliferation, differentiation and apoptosis (17-20). They act as either oncogenes or tumor suppressors in various types of human cancers including breast cancer $(21,22)$.

In our previous study, we found that TRAF2 is upregulated in breast cancer (23) and regulates cell apoptosis and proliferation in breast cancer cell lines MCF-7 and MDA-MB-231 (24). We speculated that aberrant expression of certain miRNAs contributes to overexpression of the TRAF2 gene in breast cancer.

In the present study, we identified miR-502-5p as a direct regulator of TRAF2 in breast cancer. We also confirmed that miR-502-5p plays a suppressive role in breast cancer through targeting oncogenic TRAF2, suggesting that miR-502-5p may be a useful biomarker for the diagnosis and therapy of human breast cancer.

\section{Materials and methods}

Patient tissues and cell lines. Breast cancer and paired normal tissues were obtained from 30 breast cancer patients at the First Affiliated Hospital of China Medical University between January 2010 and July 2012 with informed consent. The study was approved by the First Affiliated Hospital Ethics Review Committee. Verification of the specimens was 
performed by a pathologist, and the samples were immediately frozen at $-80^{\circ} \mathrm{C}$ after being removed from the patients. The human normal breast cell line MCF-10A and two human breast cancer cell lines MCF-7 and MDA-MB-231 were obtained from the American Type Culture Collection (ATCC; Manassas, VA, USA). The HEK293 (embryonic kidney) cell line was purchased from the Cell Biology Institute of Shanghai, Chinese Academy of Science (Shanghai, China).

Cell culture. MCF-10A cells were cultured in DMEM/F12 (1:1) (HyClone, Logan, UT, USA) supplemented with 5\% equine serum (HyClone), $10 \mu \mathrm{g} / \mathrm{ml}$ insulin and $20 \mathrm{ng} / \mathrm{ml}$ EGF. MDA-MB-231 cells were cultured in L15 (HyClone) supplemented with 10\% FBS (HyClone) and 100 units of penicillin-streptomycin. MCF-7 and HEK293 cells were routinely cultured in DMEM supplemented with $10 \%$ FBS and 100 units of penicillin-streptomycin. All of the cell lines were maintained at $37^{\circ} \mathrm{C}$ with $5 \% \mathrm{CO}_{2}$ in a humidified incubator.

Gene transfection. Small RNAs including a mimic negative control, miR-502-5p mimic, inhibitor negative control and miR-502-5p inhibitor were from Shanghai Genepharma Co., Ltd. (Shanghai, China). MCF-7 and MDA-MB-231 cells were transfected with the miRNA duplex at a final concentration of $20 \mathrm{nM}$ using Lipofectamine ${ }^{\mathrm{TM}} 2000$ in accordance with the manufacturer's procedure. In addition, a TRAF2 small interfering RNA (siRNA) designed and synthesized by Shanghai Genepharma and the hTRAF2pLPCX-HA-Flag/P874 plasmid (Addgene plasmid 20229) were purchased from Addgene (Cambridge, CA, USA) and were transfected into MCF-7 cells. The sequences of the TRAF2 siRNA were 5'-GGA CCA AGA CAA GAU UGA ATT-3' (sense) and 5'-UUC AAU CUU GUC UUG GUC CTT-3' (antisense).

Detection of transcriptional expression. Total RNA was extracted from the specimens and cell lines using TRIzol (Takara Bio, Dalian, China) according to the manufacturer's instructions. microRNA was separated using a miRcute miRNA isolation kit (Tiangen Biotech, Bejing, China) and then measured by reading the absorbance at OD260/280 nm. qRT-PCR was carried out using the ABI 7500 Real-Time PCR system (Applied Biosystems, Foster City, CA, USA) to test the expression of miR-502-5p in the breast cancer tissues and cell lines. For the detection of mature miR-502-5p, reverse transcription and quantitative PCR were performed using the One Step PrimeScript miRNA cDNA Synthesis kit (Takara) and SYBR $^{\circledR}$ Premix Ex Taq ${ }^{\mathrm{TM}}$ II (Takara). U6 small nuclear RNA (snRNA) expression was assayed for normalization. A miR502-5p specific primer and a universal reverse primer RTQ-UNIr were used for the amplification. Primer sequences for miR-502-5p and RTQ-UNIr were: 5'-ATC CTT GCT ATC TGG GTG CTA-3' and 5'-CGA ATT CTA GAG CTC GAG GCA GGC GAC ATG GCT GGC TAG TTA AGC TTG GTA CCG AGC TCG GAT CCA CTA GTC C(T)-3', respectively. Primer sequences for U6 were: F-5'-CTC GCT TCG GCA GCA CA-3' and R-5'-AAC GCT TC A CGA ATT TGC GT-3'. The PCR conditions for miR-502-5p and U6 snRNA consisted of $95^{\circ} \mathrm{C}$ for $30 \mathrm{sec}$, followed by 40 cycles of $95^{\circ} \mathrm{C}$ for $5 \mathrm{sec}$ and $60^{\circ} \mathrm{C}$ for $34 \mathrm{sec} . \Delta \mathrm{Ct}$ was calculated by subtracting the $\mathrm{Ct}$ of U6 mRNA from the $\mathrm{Ct}$ of the RNAs of interest. $\Delta \Delta \mathrm{Ct}$ was then calculated by subtracting the $\Delta \mathrm{Ct}$ of the negative control from the $\Delta \mathrm{Ct}$ of the samples. The fold-change in microRNA was calculated according to the equation $R Q=2^{-\Delta \Delta C t}$.

Flow cytometry-based apoptosis assay. Cells were grown in 6-well plates to $\sim 60 \%$ confluency and transiently transfected with miRNAs, TRAF2 siRNA and the hTRAF2pLPCX-HAFlag/P874 plasmid, respectively. The cells were digested and collected at $48 \mathrm{~h}$ post-transfection, and then washed with $1 \mathrm{X}$ PBS twice. For detection of apoptosis, the cells were treated by Annexin V-EGFP apoptosis detection kit according to the manufacturer's instructions (Nanjing KeyGen Biotech. Co., Ltd., Nanjing, China) and then analyzed with a flow cytometer (FACScalibur; Becton-Dickinson, Franklin Lakes, NJ, USA).

In vitro MTT and colony formation assays. For the MTT assay, $4-5 \times 10^{3}$ of the MCF-7 and MDA-MB-231 cells at $24 \mathrm{~h}$ post-transfection were plated into 96 -well plates. Cells were then cultured for 1, 2, 3, 4 and 5 days, respectively. The absorbance at $570 \mathrm{~nm}$ was measured after incubation of the cells with $100 \mu \mathrm{l}$ sterile MTT dye $(0.5 \mathrm{mg} / \mathrm{ml}$; Sigma) for $4 \mathrm{~h}$ at $37^{\circ} \mathrm{C}$ and $150 \mu \mathrm{l}$ DMSO for $15 \mathrm{~min}$. The cell growth curve was then constructed using OD570 $\mathrm{nm}$ as the ordinate axis. In the colony formation assay, $3-5 \times 10^{3}$ of the MCF-7 and MDA-MB-231 cells at $24 \mathrm{~h}$ post-transfection were seeded in 60-mm Petri dishes and allowed to grow until visible colonies formed. After 10-12 days, the colonies were fixed with methanol, stained with hematoxylin and scored using a microscope.

Luciferase reporter assay. Prediction of TRAF2 as a target of miR-502-5p was made with TargetScan (http://www. targetscan.org/), miRanda (http://cbio.mskcc.org/cgi-bin/ mirnaviewer $/$ mirnaviewer. pl?type $=\mathrm{miRanda}$ ) and $\mathrm{miRDB}$ (http://mirdb.org/miRDB/), respectively. Wild-type pGL3TRAF2-3'UTR (wt-pGL3-TRAF2-3'UTR) and mutant pGL3-TRAF2-3'UTR (mut-pGL3-TRAF2-3'UTR) plasmids were obtained from Shanghai Genechem Co., Ltd. (Shanghai, China). The HEK293 and MCF-7 cells seeded in 96-well plates in triplicate were cotransfected with wt-pGL3-TRAF23'UTR or mut-pGL3-TRAF2-3'UTR and miRNA-502-5p mimic or mimic negative control using Lipofectamine ${ }^{\mathrm{TM}}$ 2000 in accordance with the manufacturer's procedure. The pRL-TK (Promega Corp., Madison, WI, USA) was transfected as a normalization control. Cells were collected at $24 \mathrm{~h}$ posttransfection, and luciferase activity was then measured using a dual-luciferase reporter assay kit (Promega) and recorded by a chemiluminescence meter (Promega).

Western blot assay. Protein was extracted from the cells at $48 \mathrm{~h}$ post-transfection using a protein extraction reagent (Beyotime Institute of Biotechnology, Shanghai, China), and the protein concentration was measured using the BCA protein assay kit (Beyotime, USA). Extracts $(50 \mu \mathrm{g})$ were separated on $10 \%$ SDS-PAGE and transferred to a PVDF membrane. The membrane was then blocked with 5\% non-fat milk and incubated with the anti-TRAF2 antibody (1:200 dilution; BD Biosciences, Franklin Lakes, NJ, USA) followed by horseradish peroxidase-conjugated antibody (1:2,000 dilution; Zhongshan Golden Bridge Biotechnology Co., Ltd., Beijing, China). Detection was performed by enhanced chemiluminescence 


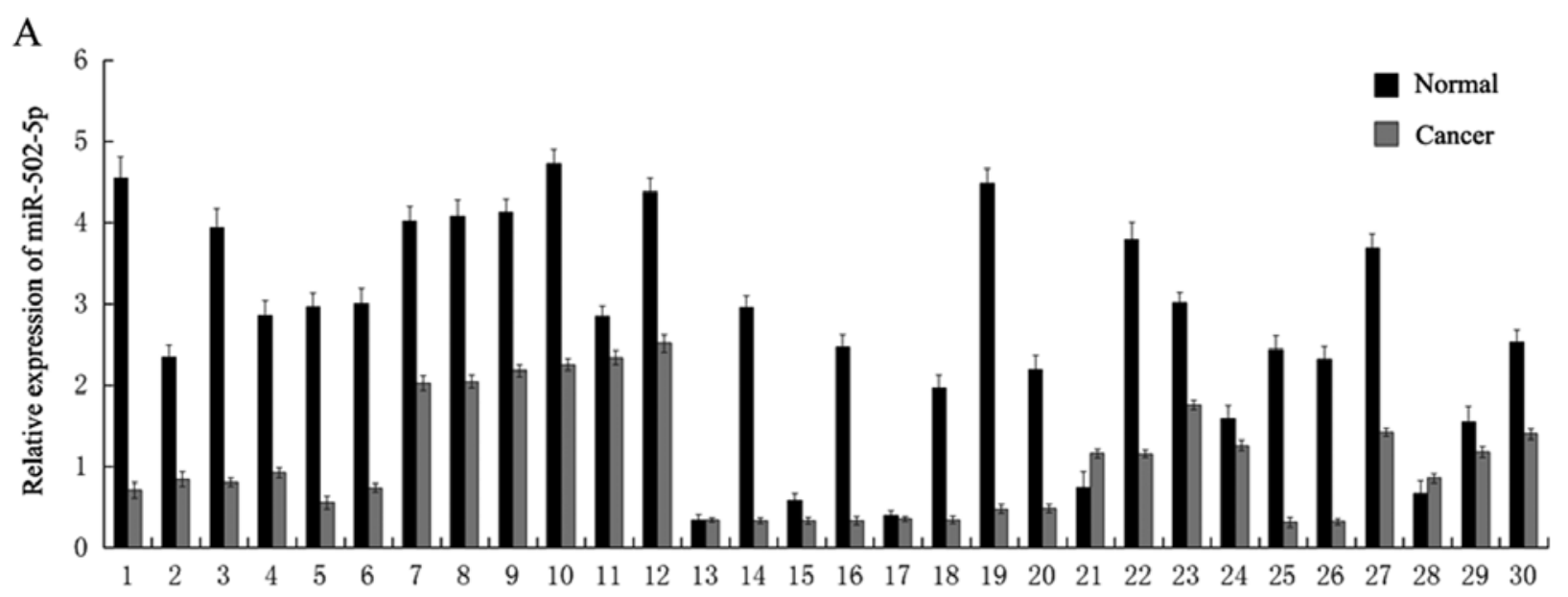

B

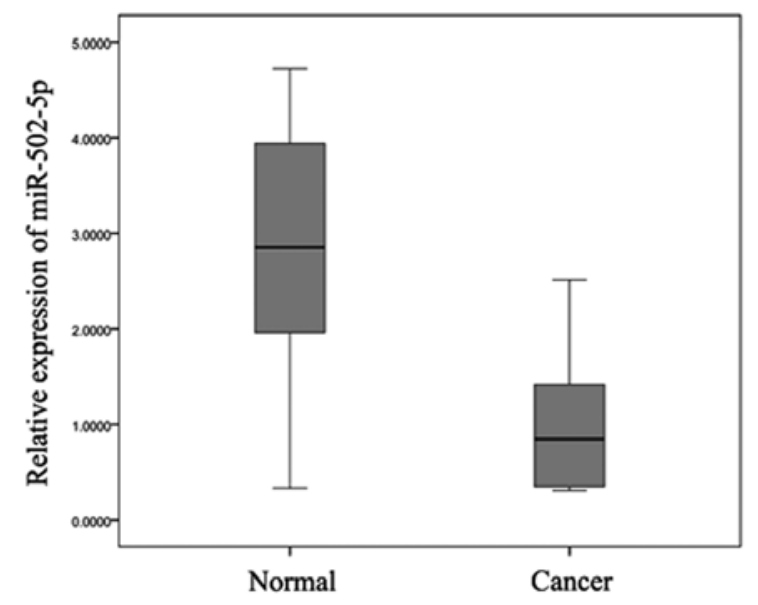

C

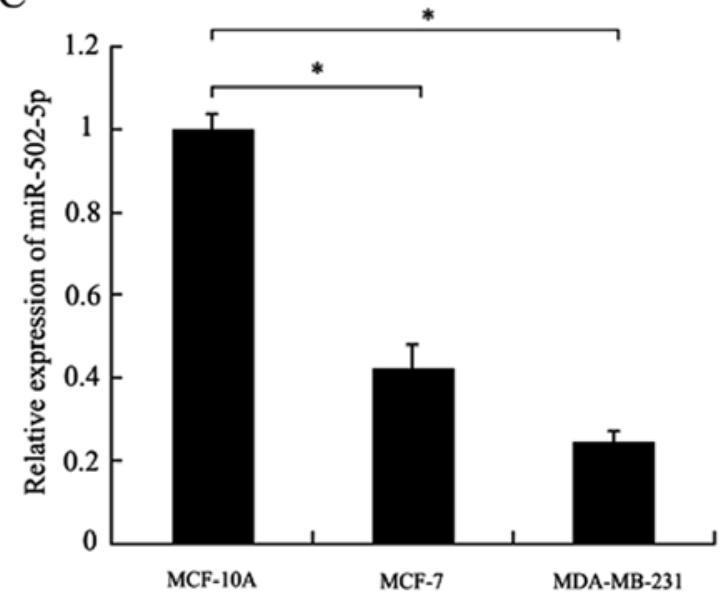

Figure 1. miR-502-5p is significantly downregulated in breast cancer tissues and cell lines. (A) Paired bar chart represents the expression of miR-502-5p in 30 breast cancer tissues and paired normal tissues as determined by qRT-PCR. U6 was used as an internal control. (B) Boxplot indicates miR-502-5p expression in breast cancer and paired normal tissues. (C) miR-502-5p expression levels in breast cancer cell lines MCF-7 and MDA-MB-231 and non-malignant breast

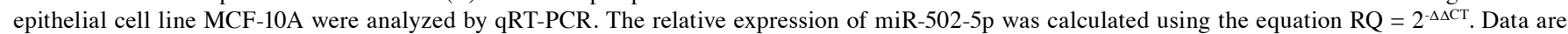
presented as means \pm SD from three independent experiments. ${ }^{*} \mathrm{P}<0.05$.

(ECL) using a Western blotting immunological reagent (Santa Cruz Biotechnology) according to the manufacturer's instructions. $\beta$-actin and GAPDH were used as internal controls and determined following the same procedure as above.

Statistical analysis. Data were subjected to statistical analysis using the SPSS 17.0 software and are shown as means \pm SD. Each experiment was performed for a minimum of three times. For qRT-PCR, the expression level of miR-502-5p was $\log 2$ transformed in breast cancer and paired normal tissues, respectively. A paired-samples t-test was used to analyze differences in miR-502-5p expression between the breast cancer and paired normal tissues. The results of the cell-based experiments were analyzed by an independent sample t-test and one-way ANOVA. $\mathrm{P}<0.05$ was considered to indicate a statistically significant result.

\section{Results}

miR-502-5p is lowly expressed in breast cancer tissues and cell lines. We detected expression of miR-502-5p by
qRT-PCR in 30 breast cancer tissue samples with paired normal tissues. Compared with the normal tissues, the expression of miR-502-5p (26/30) was significantly downregulated in the breast cancer tissues (Fig. 1A). Downregulation of miR-502-5p is also shown in the boxplot (Fig. 1B). We then examined miR-502-5p expression in the breast cancer cell lines MCF-7 and MDA-MB-231 along with the non-malignant breast epithelial cell line MCF-10A. miR-502-5p expression was lower in the MCF-7 and MDA-MB-231 cells than that in the MCF-10A cells (Fig. 1C).

miR-502-5p promotes early apoptosis and inhibits proliferation in breast cancer cells. To investigate the impact of miR-502-5p on the apoptosis and proliferation of breast cancer cells, we evaluated the transfection efficiency in MCF-7 and MDA-MB-231 cells by qRT-PCR. At $48 \mathrm{~h}$ post-transfection, the expression levels of miR-502-5p transfected with miR-502-5p mimic and its inhibitor in MCF-7 and MDA-MB-231 cells were significantly increased and decreased respectively, when compared to the level in the controls, (Fig. 2A). Flow cytometric assay results indicated that the percentages of 

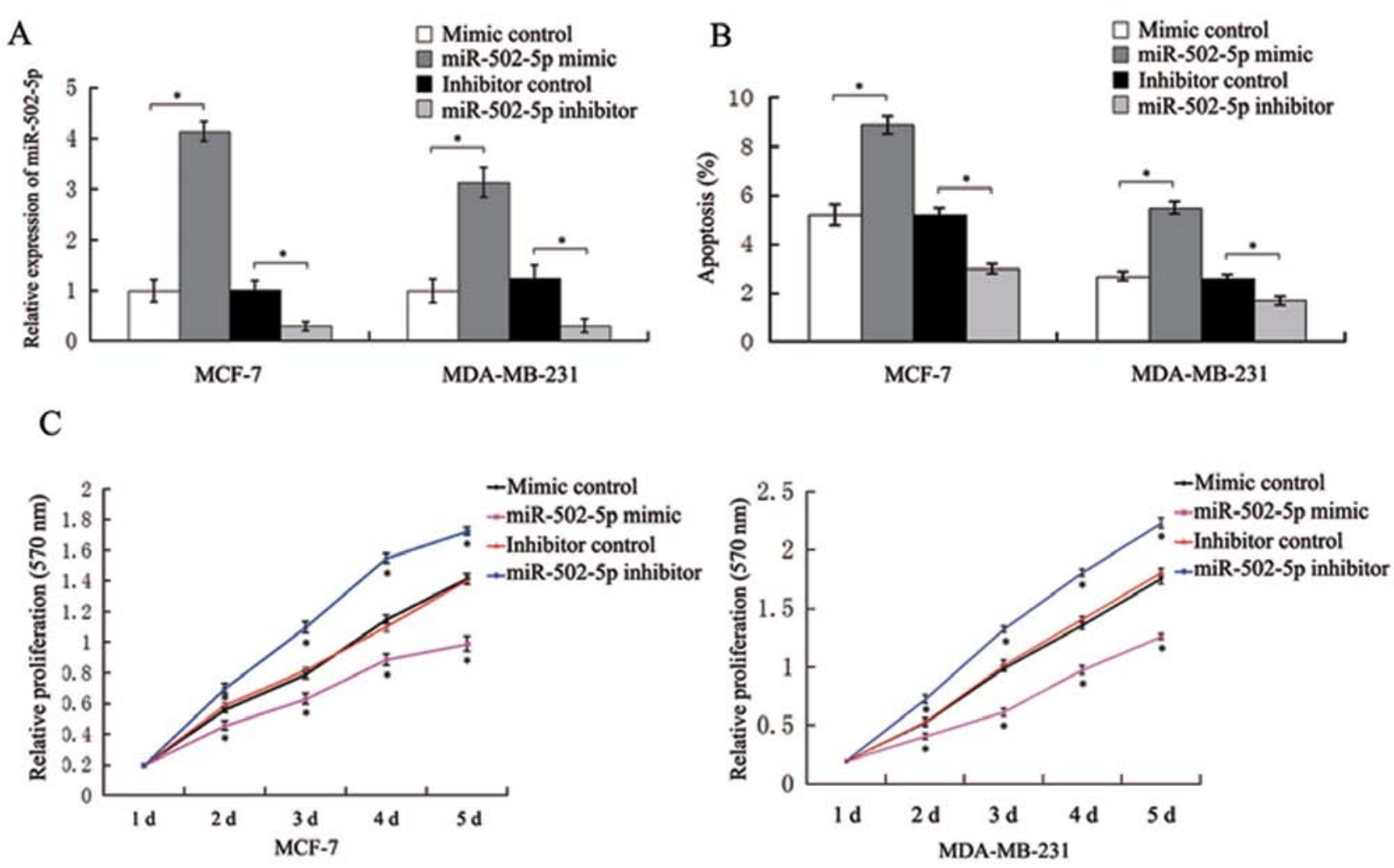

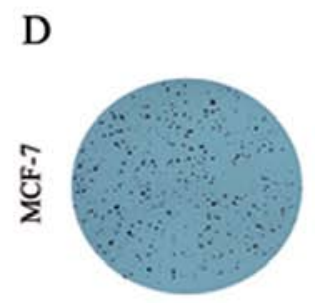

Mimic control

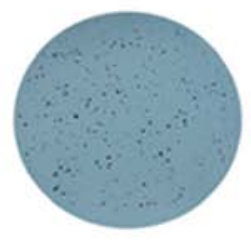

miR-502-5p mimic

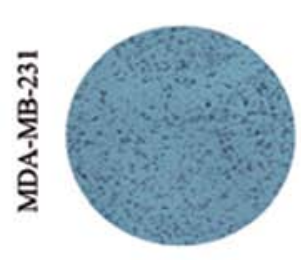

Mimic control

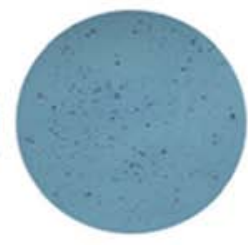

miR-502-5p mimic

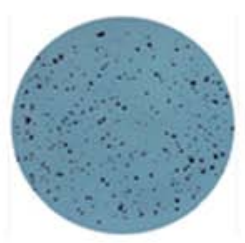

Inhibitor control

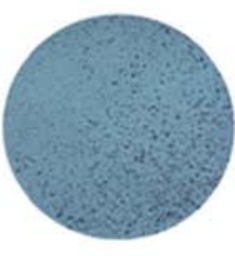

Inhibitor control

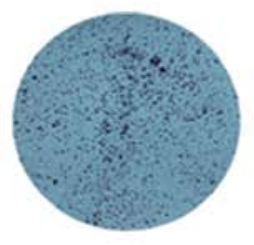

miR-502-5p inhibitor

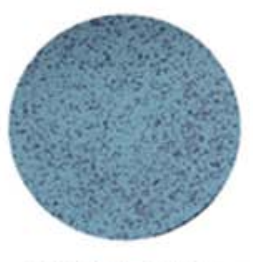

miR-502-5p inhibitor
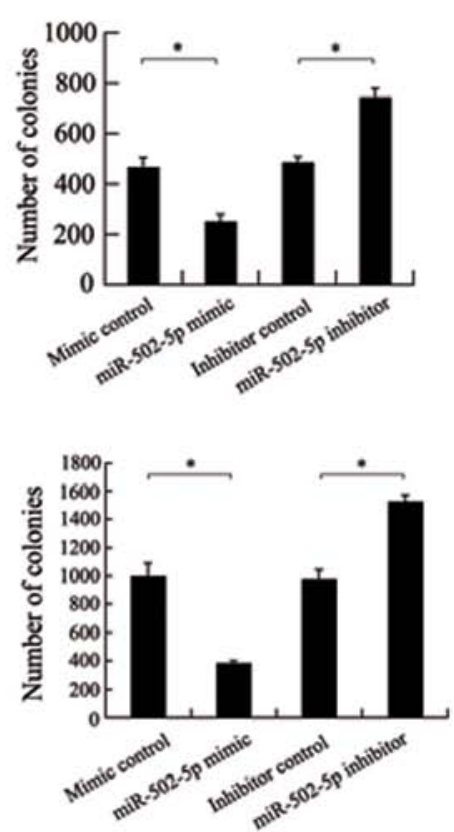

Figure 2. miR-502-5p promotes early apoptosis and inhibits proliferation in breast cancer cells. MCF-7 and MDA-MB-231 cells were transfected with the mimic control, miR-502-5p mimic, inhibitor control and the miR-502-5p inhibitor, and were then subjected to early apoptosis and proliferation assays after $48 \mathrm{~h}$. (A) The expression of miR-502-5p after transfection in MCF-7 and MDA-MB-231 cells as determined by qRT-PCR. U6 was used as an internal control. (B) Effect of miR-502-5p on early apoptosis in MCF-7 and MDA-MB-231 cells as determined by flow cytometry. (C) Effect of miR-502-5p on cell viability in MCF-7 and MDA-MB-231 cells as determined by MTT assay. (D) Effect of miR-502-5p on cell proliferation in MCF-7 and MDA-MB-231 cells as determined by colony formation assay. Data are presented as means \pm SD from three independent experiments. ${ }^{*} \mathrm{P}<0.05$.

early apoptotic MCF-7 and MDA-MB-231 cells in the miR502-5p mimic or its inhibitor group were significantly higher or lower, respectively, than the percentage in the control groups (Fig. 2B). Meanwhile, no significant difference in the cell cycle was observed in the cells transfected with the miR-502-5p mimic or its inhibitor (data not shown), indicating that miR-502-5p does not affect the cell cycle progression in MCF-7 and MDA-MB-231 cells. MTT assay results demonstrated that the miR-502-5p mimic or its inhibitor significantly suppressed or enhanced, respectively, the cell growth of MCF-7 and MDA-MB-231 when compared to the controls (Fig. 2C). Colony formation assay results revealed that the 
A

\author{
Position 452-459 of TRAF2 3' UTR \\ 5' ...CTCCTGCTGGCCAGAGCAAGGAA... \\ miR-502-5p \\ 3' AUCGUGGGUCUAUCGUUCCUA \\ Position 452-459 of mutated TRAF2 3' UTR \\ 5' ...СTCCTGCTGGCCAGATACCTTCA... \\ miR-502-5p $\quad X \times X \times X \times X$ \\ 3' AUCGUGGGUCUAUCGUUCCUA
}

B
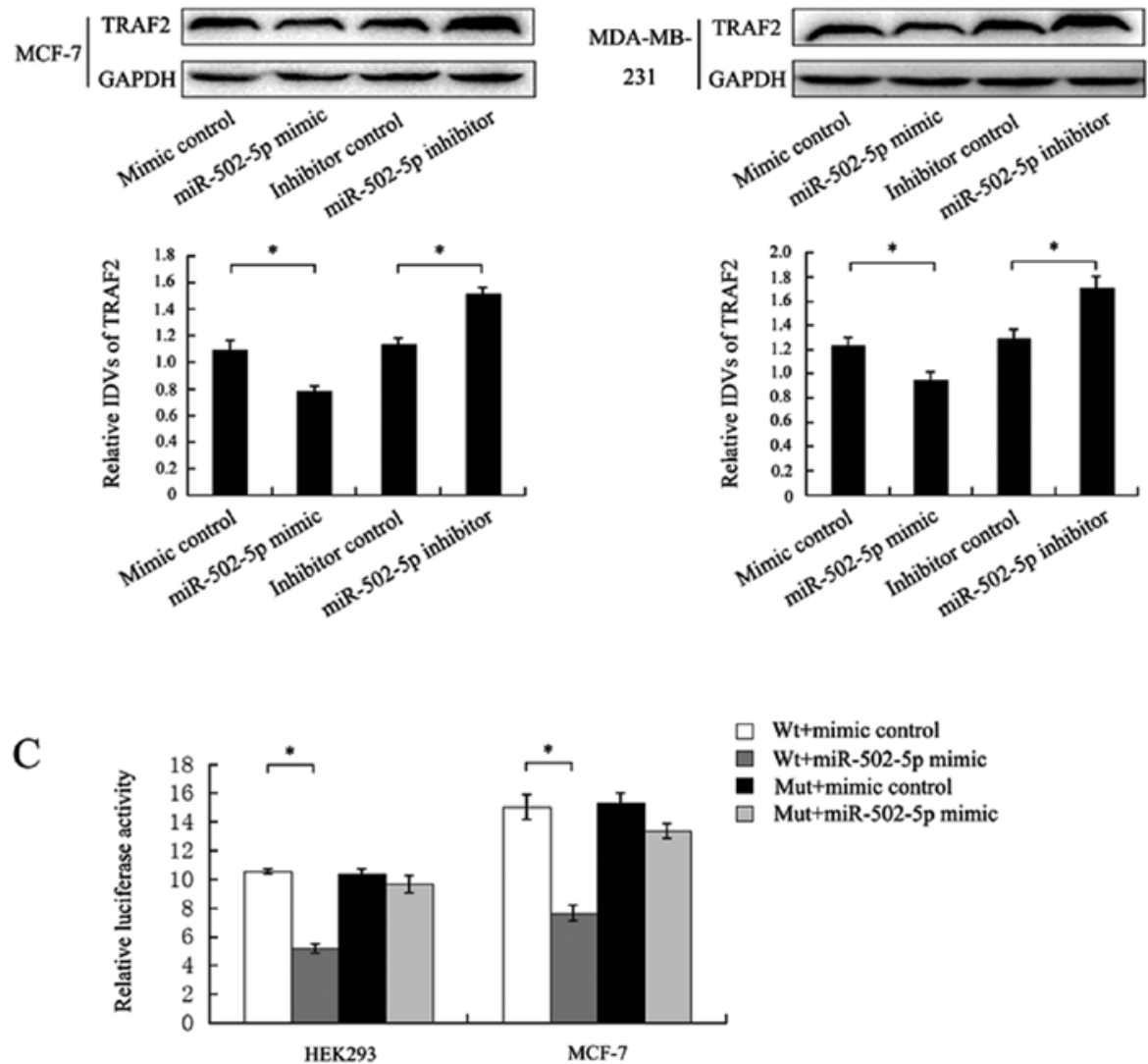

Figure 3. TRAF2 is a direct target of miR-502-5p. (A) Potential binding site of miR-502-5p with the wild-type and mutant 3'-UTR of TRAF2. (B) Effect of miR-502-5p on TRAF2 protein level in breast cancer cells. MCF-7 and MDA-MB-231 cells were transfected with the mimic control, miR-502-5p mimic, inhibitor control and the miR-502-5p inhibitor. After $48 \mathrm{~h}$, cellular protein was extracted and subjected to western blot assay for TRAF2. GAPDH was used as an internal control. (C) Direct binding of miR-502-5p to TRAF2. Luciferase reporter assay revealed that miR-502-5p suppressed TRAF2 3'-UTR luciferase activity in HEK293 and MCF-7 cells. Renilla luciferase was normalized to firefly luciferase, and data are presented as means \pm SD from three independent experiments. ${ }^{*} \mathrm{P}<0.05$. Wt, wild-type; Mut, mutant.

miR-502-5p mimic or its inhibitor significantly suppressed or promoted, respectively, the colony formation in the MCF-7 and MDA-MB-231 cells when compared to the controls (Fig. 2D). The above results suggest that miR-502-5p plays a suppressive role in breast cancer cells.

$T R A F 2$ is a direct target of miR-502-5p. As shown in Fig. 3A, the 3'-UTR of TRAF2 was found to contain a predicted miR-502-5p binding site located at 452-459 nt. Western blot assay results showed that the miR-502-5p mimic or its inhibitor significantly decreased or increased, respectively, the TRAF2 protein levels when compared to the levels in the contros MCF-7 and MDA-MB-231 cells (Fig. 3B), implying that $\mathrm{miR}-502-5 \mathrm{p}$ regulates the expression of TRAF2. To validate the direct binding of TRAF2 to miR-502-5p, we constructed wild-type and mutant TRAF2 3'-UTR luciferase reporter vectors. Luciferase reporter assay results indicated that miR-502-5p significantly inhibited the luciferase activity when co-transfected with the wild-type vector, but not when co-transfected with the mutant vector in HEK293 cells (Fig. 3C). We also obtained the same results in MCF-7 cells (Fig. 3C). Collectively, these results implied that TRAF2 is one of the target genes of miR-502-5p and is suppressed by miR-502-5p. 
A

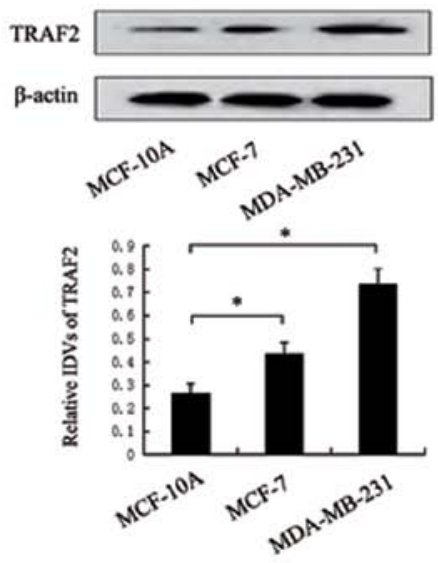

$\mathrm{C}$

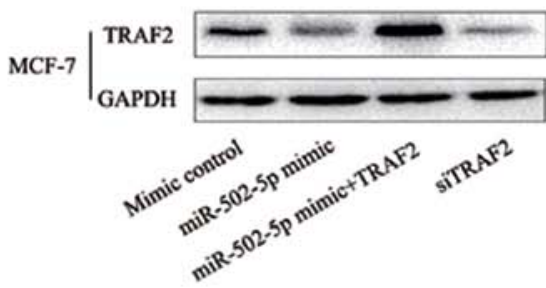

B
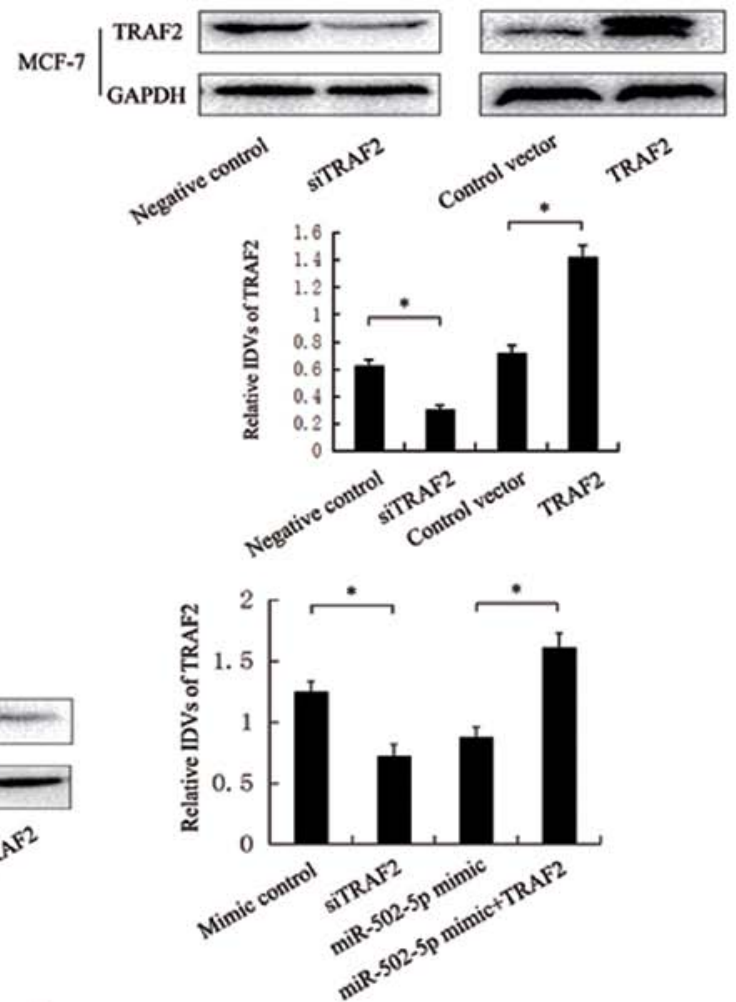

$\mathrm{D}$

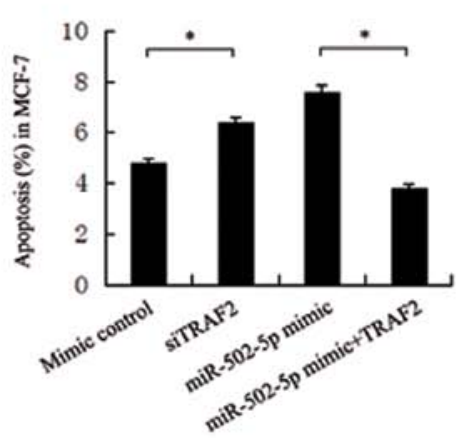

$\mathrm{F}$

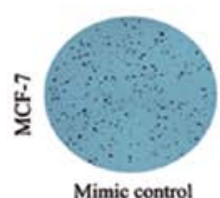

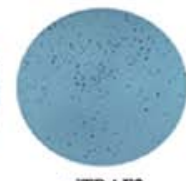

SiTRAF2
E

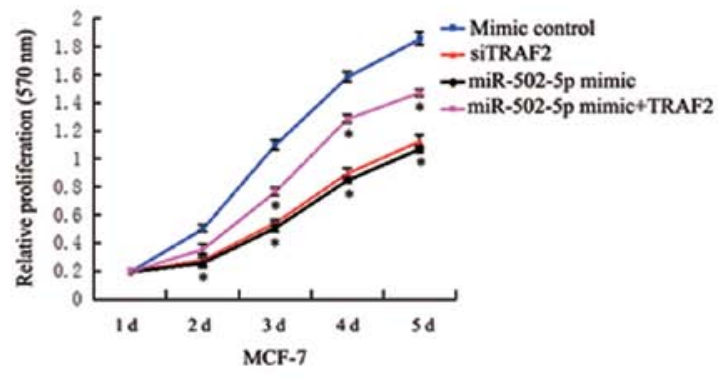

Figure 4. miR-502-5p regulates breast cancer cell early apoptosis and proliferation by targeting TRAF2. (A) TRAF2 protein levels in breast cancer cell lines MCF-7 and MDA-MB-231 and non-malignant breast epithelial cell line MCF-10A as determined by western blot analysis. $\beta$-actin was used as an internal control. (B) Western blot assay of TRAF2 following transfection of TRAF2 siRNA and the TRAF2 plasmid in MCF-7 cells. GAPDH was used as an internal control. (C) Western blot assay of TRAF2 following transfection of mimic control, TRAF2 siRNA, miR-502-5p mimic and miR-502-5p mimic plus TRAF2 plasmid in MCF-7 cells. GAPDH was used as an internal control. (D) Effect of TRAF2 siRNA and co-transfection of miR-502-5p mimic plus TRAF2 plasmid on early apoptosis in MCF-7 cells as determined by flow cytometry. (E) Effect of TRAF2 siRNA and co-transfection of miR-502-5p mimic plus TRAF2 plasmid on cell viability in MCF-7 cells as determined by MTT assay. (F) Effect of TRAF2 siRNA and co-transfection of miR-502-5p mimic plus TRAF2 plasmid on cell proliferation in MCF-7 cells as determined by colony formation assay. Data are presented as means \pm SD from three independent experiments. ${ }^{*} \mathrm{P}<0.05$.

miR-502-5p regulates early apoptosis and proliferation of breast cancer cells by targeting TRAF2. Western blot assay results revealed that TRAF2 protein levels were significantly higher in MCF-7 and MDA-MB-231 cells than that in the MCF-10A cells (Fig. 4A). On the contrary, miR-502-5p expression levels were significantly lower in 
MCF-7 and MDA-MB-231 cells than that in the MCF-10A cells (Fig. 1C), suggesting the negative correlation of miR502-5p with TRAF2 in breast cancer and normal cells. To confirm whether miR-502-5p regulates early apoptosis and proliferation of breast cancer cells by targeting TRAF2, TRAF2 siRNA and the TRAF2 plasmid were used to induce the knockdown and overexpression, respectively, of TRAF2 expression. Western blot assay results revealed that TRAF2 siRNA significantly inhibited the endogenous TRAF2 expression when compared to the expression in the control group, and the opposite result was found when the TRAF2 plasmid was tranfected into the MCF-7 cells (Fig. 4B). We also found that the TRAF2 plasmid significantly restored the TRAF2 protein level in MCF-7 cells when co-transfected with the miR-502-5p mimic (Fig. 4C). Flow cytometric assay results indicated that knockdown of TRAF2 significantly increased the early apoptosis of breast cancer cells when compared to the control group in the MCF-7 cells (Fig. 4D). MTT and colony formation assay results showed that knockdown of TRAF2 significantly inhibited cell viability and colony formation when compared to the control group in the MCF-7 cells (Fig. 4E and F). Overexpression of TRAF2 partly rescued the effects of the miR-502-5p mimic in regard to early apoptosis, cell viability and colony formation in the MCF-7 cells when co-transfected with the miR-502-5p mimic (Fig. 4D-F). These results suggest that miR-502-5p may be crucial in the regulation of cell apoptosis and proliferation through targeting oncogenic TRAF2.

\section{Discussion}

Breast cancer is the most commonly diagnosed malignancy and is the leading cause of cancer-related mortality among women worldwide (25). Therefore, searching for valuable biomarkers for the early diagnosis and treatment of breast cancer remains a focus of breast cancer research. Recent studies indicate that miRNAs are promising molecular markers for diseases including cancer due to their stability in human tissues particularly in blood and urine $(26,27)$.

miRNA-502 was found to be downregulated in colon cancer tissues and inhibits autophagy, colon cancer cell growth and cell-cycle progression, suggesting that it may be a novel candidate for miR-502-based therapeutic strategies (28). However, little is known concerning the role of miR-502-5p in other types of cancers including breast cancer. In the present study, we found that the miR-502-5p expression level was significantly decreased in breast cancer tissues and cell lines and that exogenous miR-502-5p promoted early apoptosis and suppressed the growth and colony formation of breast cancer cells, but had no effect on the breast cancer cell cycle. Both studies imply that miR-502 plays a suppressive role in colon and breast cancer.

In the present study, miR-502-5p was predicted to be a potential upstream regulator of TRAF2. miR-502-5p was negatively correlated with TRAF2 expression in breast cancer cells, further implying that TRAF2 is a candidate target of miR-502-5p. The miR-502-5p mimic significantly decreased the luciferase activity when it was cotransfected with wt-pGL3-TRAF2-3'UTR compared to the controls, indicating that miR-502-5p directly binds to TRAF2. In addition to TRAF2, SET8 was previously confirmed as another direct target of miR-502-5p (29).

Similar to the role of the miR-502-5p mimic in the MCF-7 cells, we also found that TRAF2 siRNA significantly enhanced the early apoptosis and suppressed cell viability and colony formation. Meanwhile, overexpression of TRAF2 decreased the effects of the miR-502-5p mimic in the MCF-7 cells to some extent. Based on the above results, we speculate that miR-502-5p plays a tumor-suppressive role in breast cancer by directly targeting TRAF2.

We previously found that TRAF2 regulates cell proliferation and apoptosis by activating NF- $\mathrm{B}$ nuclear transcription by directly binding to TRAF4 in breast cancer (24). Therefore, we conclude that miR-502-5p serves as a tumor-suppressor gene via regulation of the interaction of TRAF2 and TRAF4, leading to inhibition of the NF- $\mathrm{B}$-mediated signaling pathway in breast cancer. This suggests that miR-502-5p may provide a promising therapeutic strategy for breast cancer treatment.

\section{Acknowledgements}

The present study was supported by the Research Fund for the Doctoral Program of Higher Education (20112104110017).

\section{References}

1. Chung JY, Park YC, Ye $\mathrm{H}$ and Wu H: All TRAFs are not created equal: common and distinct molecular mechanisms of TRAFmediated signal transduction. J Cell Sci 115: 679-688, 2002.

2. Xia ZP and Chen ZJ: TRAF2: a double-edged sword? Sci STKE 2005: pe7, 2005.

3. Arch RH and Thompson CB: 4-1BB and Ox40 are members of a tumor necrosis factor (TNF)-nerve growth factor receptor subfamily that bind TNF receptor-associated factors and activate nuclear factor $\kappa$ B. Mol Cell Biol 18: 558-565, 1998.

4. Duckett CS, Gedrich RW, Gilfillan MC and Thompson CB: Induction of nuclear factor kappaB by the CD30 receptor is mediated by TRAF1 and TRAF2. Mol Cell Biol 17: 1535-1542, 1997.

5. Hsu H, Shu HB, Pan MG and Goeddel DV: TRADD-TRAF2 and TRADD-FADD interactions define two distinct TNF receptor 1 signal transduction pathways. Cell 82: 299-308, 1996.

6. Reinhard C, Shamoon B, Shyamala V and Williams LT: Tumor necrosis factor $\alpha$-induced activation of c-jun $\mathrm{N}$-terminal kinase is mediated by TRAF2. EMBO J 16: 1080-1092, 1997.

7. Rothe M, Sarma V, Dixit VM and Goeddel DV: TRAF2-mediated activation of NF-kappa B by TNF receptor 2 and CD40. Science 269: 1424-1427, 1995.

8. Takeuchi M, Rothe $\mathrm{M}$ and Goeddel DV: Distinct domains for nuclear factor- $\kappa \mathrm{B}$ activation and association with tumor necrosis factor signaling proteins. J Biol Chem 271: 19935-19942, 1996.

9. Liu ZG, Hsu H, Goeddel DV and Karin M: Dissection of TNF receptor 1 effector functions: JNK activation is not linked to apoptosis while $\mathrm{NF}-\kappa \mathrm{B}$ activation prevents cell death. Cell 87: 565-576, 1996.

10. Natoli G, Costanzo A, Ianni A, Templeton DJ, Woodgett JR, Balsano C and Levrero M: Activation of SAPK/JNK by TNF receptor 1 through a noncytotoxic TRAF2-dependent pathway. Science 275: 200-203, 1997.

11. Cao Z, Henzel WJ and Gao X: IRAK: a kinase associated with the interleukin-1 receptor. Science 271: 1128-1131, 1996.

12. Song HY, Regnier CH, Kirschning CJ, Goeddel DV and Rothe M: Tumor necrosis factor (TNF)-mediated kinase cascades: bifurcation of nuclear factor-kappaB and c-jun N-terminal kinase (JNK/ SAPK) pathways at TNF receptor-associated factor 2. Proc Natl Acad Sci USA 94: 9792-9796, 1997.

13. Bartel DP: MicroRNAs: genomics, biogenesis, mechanism and function. Cell 116: 281-297, 2004.

14. Jing Q, Huang S, Guth S, et al: Involvement of microRNA in AU-rich element-mediated mRNA instabiliby. Cell 120: 623-634, 2005. 
15. Bartel DP: MicroRNAs: target recognition and regulatory functions. Cell 136: 215-233, 2009.

16. Friedman RC, Farh KK, Burge CB and Bartel DP: Most mammalian mRNAs are conserved targets of microRNAs. Genome Res 19: 92-105, 2009

17. He L and Hannon GJ: MicroRNAs: small RNAs with a big role in gene regulation. Nat Rev Genet 5: 522-531, 2004.

18. Lecellier CH, Dunoyer P, Arar K, et al: A cellular microRNA mediates antiviral defense in human cells. Science 308: 557-560, 2005.

19. Sullivan CS, Grundhoff AT, Tevethia S, Pipas JM and Ganem D: SV40-encoded microRNAs regulate viral gene expression and reduce susceptibility to cytotoxic T cells. Nature 435: 682-686, 2005.

20. Pallante P, Visone R, Ferracin M, et al: MicroRNA deregulation in human thyroid papillary carcinomas. Endocr Relat Cancer 13: 497-508, 2006

21. Zhang B, Pan X, Cobb GP and Anderson TA: microRNAs as oncogenes and tumor suppressors. Dev Biol 302: 1-12, 2007.

22. Chen CZ: MicroRNAs as oncogenes and tumor suppressors. N Engl Med 353: 1768-1771, 2005.

23. Wang S, Mi X, Liu N, Fang C, Teng Y and Zhao R: Expression of TRAF2 and its relationship with invasion in breast cancer. J China Med Univ 37: 5, 2008.
24. Zhang X, Wen Z, Sun L, Wang J, Song M, Wang E and Mi X: TRAF2 regulates the cytoplasmic/nuclear distribution of TRAF4 and its biological function in breast cancer cells. Biochem Biophys Res Commun 436: 344-348, 2013.

25. Haghighat S, Akbari ME, Ghaffari S and Yavari P: Standardized breast cancer mortality rate compared to the general female population of Iran. Asian Pac J Cancer Prev 13: 5525-5528, 2012.

26. Blondal T, Jensby Nielsen S, Baker A, Andreasen D, Mouritzen P, Wrang Teilum $M$ and Dahlsveen IK: Assessing sample and miRNA profile quality in serum and plasma or other biofluids. Methods 59: S1-S6, 2013.

27. Lorenzen JM and Thum T: Circulating and urinary microRNAs in kidney disease. Clin J Am Soc Nephrol 7: 1528-1533, 2012.

28. Zhai H, Song B, Xu X, Zhu W and Ju J: Inhibition of autophagy and tumor growth in colon cancer by miR-502. Oncogene 32 : 1570-1579, 2013.

29. Ding C, Li R, Peng J, Li S and Guo Z: A polymorphism at the miR-502 binding site in the 3' untranslated region of the SET8 gene is associated with the outcome of small-cell lung cancer. Exp Ther Med 3: 689-692, 2012. 